Université d'Abomey Calavi

(Bénin)

Abdel Rahamane Baba-Moussa Université d'Abomey Calavi

(Bénin)

\section{La formation initiale et continue au Bénin : entre professionnalisation et déprofessionnalisation}

\author{
Teaching function and reform of education in Benin: \\ between professionalization and de-professionalization
}

La fonction enseignante suscite au Bénin des débats entre " professionnalisation » et « déprofessionnalisation » du fait des mesures de gratuité scolaire prises en 2006 en marge des prévisions du Plan décennal de développement du secteur de l'éducation, et qui ont conduit à l'utilisation d'enseignants « communautaires » non qualifiés pour faire face aux effectifs croissants. Le présent article interroge doublement le rôle de la formation initiale et continue d'une part et l'utilisation des enseignants communautaires d'autre part, sur la professionnalisation des enseignants et la qualité de l'enseignement. L'enquête qualitative réalisée auprès de 53 élèves maîtres en formation dans les Écoles normales d'instituteurs montre qu'au-delà de l'utilisation des enseignants non qualifiés se pose plus globalement le problème de la qualité de la formation des enseignants avec une tension entre des mesures favorables à la professionnalisation et des effets relevant plutôt de la déprofessionnalisation. Ainsi, la formation initiale ne semble apporter quasiment aucune plus-value et les données disponibles sur les résultats des établissements scolaires montrent que la présence des enseignants communautaires n'est pas corrélée à la baisse de la qualité

de l'enseignement. L'article fournit ainsi un ensemble d'informations susceptibles d'aider à la prise en compte de la question enseignante dans la réforme de l'éducation.

\section{Mots-clés}

Fonction enseignante, formation initiale et continue, professionnalisation, déprofessionnalisation.

Abstract

The teaching function in Benin brings debate between "professionalization» and «de-professionalization» because of the measures of school free access, taken in 2006 outside the forecasts of the Ten-year Plan to develop the education sector, and which led to the use of «community» teachers not qualified to face the growing staff. The present article questions both the role of the initial and continuous training on one hand and the use of the community teachers on the other hand, on the professionalization of the teachers and the quality

of the education (teaching). The qualitative survey realized with 53 trainees in the teachers' training colleges for Primary school teachers shows that beyond the use of the not qualified teachers, there is more generally a problem relating to the quality of the teacher training with a tension between measures supporting the professionalization and the effects being rather like de-professionalization. So the initial training seems to bring almost no capital gain and the data available about the school results shows that the community presence of the teachers is not correlated to a sub-standard education. The paper provides a set of information that could help taking into account the teaching aspect in the reform of the education.

\section{Introduction}

L'Éducation pour tous, prônée depuis plus d'une quinzaine d'années, a favorisé dans la plupart des pays africains le phénomène de la massification scolaire. Cette dernière a plus souvent pris, selon Baba-Moussa (2006), l'allure d'une «démographisation» plutôt que d'une démocratisation puisque l'augmentation importante de l'accès ne s'est pas accompagnée de la qualité des acquis scolaires. La volonté de garantir l'éducation à tous a conduit bon nombre d'États d'Afrique occidentale à pourvoir les écoles en enseignants aux profils divers, parfois sans qualification. Une des conséquences en est la baisse de la qualité du corps enseignant avec un risque important de "déprofessionnalisation " (Perrenoud, 1996). Par ailleurs, le fait de confier l'enseignement à des tiers non enseignants suscite des interrogations face à la baisse de la qualité des apprentissages et relance le débat sur la profession enseignante. C'est cette situation que l'on envisage ici d'explorer au Bénin afin d'en tirer les informations utiles à la prise de décisions dans la perspective de la réforme de l'éducation. Des précisions conceptuelles et une présentation du contexte des enseignants du primaire permettront d'abord de cerner la problématique telle qu'elle se pose au Bénin. Ensuite, une enquête auprès de 53 élèves-maîtres en formation appuyée par des résultats d'études antérieures montreront que, si les mesures sont prises dans l'intention d'assurer la professionnalisation par l'adoption d'une formation à l'autonomie et à la réflexivité, la réalité des pratiques traduit, à l'opposé, une démarche « très directive » peu favorable aux résultats attendus. 


\section{Cadre théorique et problématique de la recherche}

Afin de pallier l'insuffisance d'enseignants, on a eu recours, dans les politiques éducatives successives au Bénin, aux enseignants non qualifiés dits «communautaires ». En 2008, le pays a opté pour le reversement des enseignants communautaires en enseignants contractuels de l'État afin de résorber le déficit en enseignants qualifiés. Si l'on tient compte de l'impact de l'utilisation des enseignants communautaires sur le ratio élèves-maître (REM), on pourrait même être tenté de conclure que leur utilisation est plutôt bénéfique. En effet, si le ratio élèves-maître n'explique pas la baisse de la qualité des acquis, il constitue une bonne source d'analyse puisque, par exemple, l'aide-mémoire de la revue sectorielle de l'éducation de mai 2016 montre une amélioration de la qualité des apprentissages entre 2011 et 2013, période où le ratio élèves-maître était d'environ 48. Cette amélioration ne s'est pas maintenue en 2014 et en 2015 où les ratios étaient respectivement à 53,1 puis à 52,4, soit une différence de 5 points en 2 ans qui traduit un lourd déficit en enseignant avec des écarts encore plus importants selon les départements. Ainsi, l'aide-mémoire souligne une aggravation chaque année depuis 2008 de l'écart entre les ratios élèves-maître (REM) des départements extrêmes : 26,4 en 2015 contre 8,7 en 2008. Cette contre-performance s'explique par l'accentuation du déficit de la dotation des écoles en enseignants : de 39,8 \% en 2010, ce déficit est passé à 47,9\% en 2015 (STP-PDDSE, 2016).

Le bas niveau des acquis scolaires peut s'expliquer par le faible niveau des enseignants en général, mais aussi par l'absence d'un dispositif de formation continue efficace. En ce qui concerne le niveau des enseignants, l'évaluation intitulée « ce que je sais de ce que j'enseigne » a permis de mesurer le savoir des enseignants avec des outils standardisés (Bahna et Houédo, 2015). Cette évaluation concluait que les résultats obtenus par les enseignants du Cours élémentaire niveau 1 et du Cours élémentaire niveau 2, qui sont souvent tenus par des communautaires et des élèves-maîtres, étaient modérés, mais aussi que des enseignants titulaires d'un diplôme professionnel enregistraient de très faibles performances : « curieusement, la faible performance la plus inquiétante $(7 \%)$ est enregistrée au niveau des enseignants titulaires du CM2 qui sont par surcroît les Directeurs d'écoles, les enseignants les plus anciens » (Bahna et Houédo, 2015). Cette même étude montre que les enseignants, agents permanents de l'État (fonctionnaires et donc ayant bénéficié d'une formation initiale qualifiante) ne se distinguent pas, du point de vue de leurs performances, des enseignants contractuels (souvent des enseignants communautaires reversés). Partant de cela, on peut déduire que rien ne permet de valider l'idée selon laquelle la baisse du niveau scolaire serait attribuable à l'utilisation des enseignants communautaires.

En ce qui concerne la formation continue organisée dans les unités pédagogiques (UP), elle devait permettre aux enseignants de disposer d'outils nécessaires pour affronter les situations de classe. Cependant, les données existantes révèlent qu'elle se déroule sans une ingénierie de la formation. En effet, au cours de ses travaux sur le fonctionnement des unités pédagogiques, réalisés à partir de l'analyse des besoins (Ardoin, 2013), Boko-vou (2015) a relevé plusieurs irrégularités. Il montre en particulier que la formation en UP est en déphasage par rapport aux besoins en formation des enseignants et qu'elle se fait en l'absence d'une démarche d'analyse des besoins. Ainsi, le formé reste bien distant de l'objet de formation. Selon Boko-vou, le modèle d'analyse des besoins a trois niveaux d'enjeux majeurs pour la formation. Il s'agit d'abord du niveau politique pour la définition des compétences nécessaires à l'exercice de la fonction enseignante, ensuite du niveau organisationnel qui permet à la formation de constituer le levier de changement et d'accompagnement des enseignants 
et enfin du niveau opérationnel qui concerne les apprentissages et le transfert des habiletés. Chaque niveau regroupe une catégorie d'acteurs, mais c'est le niveau opérationnel qui influe directement sur le développement de l'enseignant. Or, on peut constater à ce niveau un cloisonnement des fonctions dans lequel les conseillers pédagogiques et les inspecteurs peinent à associer les responsables d'unité pédagogique et les enseignants à l'élaboration des modules de formation. Ces modules sont élaborés par les conseillers pédagogiques et les inspecteurs puis remis au responsable d'unité pédagogique pour exploitation alors même qu' «il n'existe pas d'instrument spécifique conçu et destiné à l'analyse des besoins »(Boko-vou, 2015).

Il en découle une problématique spécifique que l'on peut poser ici en termes de professionnalisation et de déprofessionnalisation des enseignants. Le présent article s'intéresse à deux profils d'enseignants : ceux formés dans les écoles de formation et ceux formés sur le tas généralement appelés « enseignants communautaires ». Il s'agit de voir si l'existence de cette deuxième catégorie d'enseignants, preuve que l'entrée dans la fonction enseignante n'est pas contrôlée par les enseignants, se traduirait par des effets de déprofessionnalisation. Il s'agit aussi de voir dans quelle mesure cela pourrait expliquer la mauvaise qualité de l'enseignement constatée dans les différentes évaluations. Mais au-delà de ces aspects, c'est la formation initiale qui distingue les enseignants formés dans les Écoles normales d'instituteurs (ENI) des communautaires formés sur le tas qu'il s'agit de mettre en avant pour apprécier sa pertinence dans son contexte actuel. Nous partirons donc des concepts « professionnalisation » et « déprofessionnalisation » pour poser la problématique de recherche.

\section{Approche théorique des termes professionnalisation, déprofessionnalisation et autonomisation.}

La professionnalisation désigne «le processus par lequel une activité devient une profession libérale mue par un idéal de service » (Wittorski, 2008, p. 2). Cette définition évoque la profession comme étant un cadre d'intervention nécessitant des compétences professionnelles précises, mais semble passer sous silence l'intérêt corporatiste qui sous-tend parfois les professions en quête d'une meilleure qualité de vie. L'environnement sociopolitique actuel des professions conduit Maingari (1997) à en proposer une définition plus complexe. Pour lui, la professionnalisation renvoie tantôt à la socialisation professionnelle (comme moyen d'acquisition d'une culture et des règles de pratiques professionnelles communes), tantôt à l'identité professionnelle (en vue de l'affirmation de l'appartenance à un groupe occupationnel et de reconnaissance sociale dudit groupe), tantôt au syndicalisme (comme mode d'affirmation des droits et de défense des intérêts du groupe). Il faut noter ici l'importance de l'intériorisation des savoirs comme dimension capitale de la professionnalisation. L'intériorisation des savoirs participe à la construction des savoirs (Becker, 2004) et constitue une phase capitale de la construction identitaire. De même, le syndicalisme représente souvent la forme principale de l'organisation enseignante et vise la défense des intérêts des salariés face aux employeurs, mais un déséquilibre peut s'observer lorsque les luttes syndicales portent de façon plus importante sur la revendication des augmentations de salaires au détriment de l'amélioration de la qualité de la formation initiale. Cela peut produire des effets pervers en matière de professionnalisation.

En identifiant le processus de professionnalisation de l'enseignement à la socialisation professionnelle, Maingari considère que le milieu professionnel constitue le cadre idéal de la construction des savoirs professionnels. Il rejoint donc Altet (2000) qui estime que la professionnalisation est caractérisée par 
trois dimensions : la dimension sociale, la dimension institutionnelle et la dimension professionnelle. La dimension professionnelle concerne spécifiquement les professionnels qui se réunissent autour des questions exclusives de classe. Cette dimension met en avant l'étroitesse de la relation entre l'enseignant et sa profession. Ainsi, les stratégies développées sont de nature à rendre l'enseignant autonome, mais la professionnalisation de la fonction enseignante n'est pas un processus simple. Parfois, le rôle de l'État est défavorable à cette professionnalisation; dans d'autres cas, ce sont les professionnels eux-mêmes qui constituent un frein à la professionnalisation. Lorsque les savoirs professionnels des formateurs s'imposent aux futurs enseignants, il y a le risque de former des enseignants qui ne seront pas capables de répondre aux besoins du métier (Adékou et Baba-Moussa, 2017).

Perrenoud (1996) propose quant à lui le terme de « déprofessionnalisation » pour traduirel'accaparement de certaines composantes de la fonction enseignante par des experts appartenant à la noosphère (Chevallard, cité dans Perrenoud, 1996). Pour Perrenoud, il existe une " déprofessionnalisation » dès lors que les enseignants ne sont pas les seuls acteurs au premier plan de l'exercice de la fonction enseignante. Cette intervention d'autres acteurs, tout comme celle de l'État (Attanasso, 2010) dans l'élaboration des programmes, par exemple, réduit la notoriété de la fonction enseignante.

C'est au regard de ces éléments théoriques que nous poserons le problème de la professionnalisation de la fonction enseignante dans le contexte du Bénin au regard, d'une part, des modalités de la formation initiale et continue et, d'autre part, de l'intervention des enseignants communautaires recrutés sans formation.

\section{La professionnalisation de la fonction enseignante et ses difficultés au Bénin}

Au Bénin, les données disponibles montrent une volonté de professionnalisation de l'enseignement de par l'existence d'écoles de formation (Écoles normales d'instituteurs) régie par des textes, d'une déontologie (le guide du normalien), de diplômes qui sanctionnent la formation, de programmes de formation, etc. Cependant, le processus est confronté à des situations telles que l'intervention de l'État comme recruteur du personnel, avec comme conséquence que la corporation se trouve ainsi inféodée à l'État qui la contrôle de façon stricte, gère l'évolution des fonctionnaires et décore les plus méritants selon les procédures administratives en vigueur. De la même manière, les savoirs à enseigner ainsi que leur production sont assurés par une autre catégorie d'experts. En effet, au Bénin, à la suite de l'adoption de l'approche par compétences, la production des programmes et des guides est assurée par des équipes dont la plupart des membres n’ont pas travaillé comme enseignants. De façon générale, les réformes curriculaires opérées dans les pays de l'Afrique occidentale dont le Bénin sont souvent portées par des voix autres que celles des enseignants. Ces différents constats créent souvent une tension entre professionnalisation et déprofessionnalisation, que semblent renforcer les conditions de formation peu propices à l'autonomisation.

Le développement de l'autonomie dans l'exercice de la fonction enseignante peut être considéré comme un indicateur de la professionnalisation. Or, l'autonomisation n'est possible que lorsque l'enseignant est confronté à des situations dans lesquelles il sollicite ses propres ressources pour résoudre les problèmes auxquels il est confronté. Si les élèves-maîtres formés dans les écoles de formation d'enseignants bénéficient d'un stage sur le terrain professionnel après un an de formation, les enseignants communautaires, jusqu'en 2006, ne suivaient aucune formation avant d'enseigner. Or, le développement 
des compétences professionnelles et de l'autonomie se fait à travers les dispositifs d'accompagnement en formation initiale (Altet, 2015). L'autonomisation devient difficile lorsque l'enseignant ne peut choisir ses méthodes ou définir les stratégies propices à l'apprentissage des élèves et qu'il se voit plutôt dans l'obligation d'exécuter des formules dont il ne mesure ni la portée ni la pertinence. En effet, l'élèvemaître en formation a besoin de se convaincre de ce qu'il fait. Une intervention qui lui imposerait des recettes ne favoriserait pas cela. Ainsi, le risque résiderait dans la pression que pourrait exercer les formateurs sur les futurs enseignants dans un pays comme le Bénin où la formation des enseignants comporte un caractère prescriptif dont l'enseignant en formation ne peut se défaire que si son niveau intellectuel lui permet une prise de recul pour rejeter les prescriptions jugées non pertinentes afin de construire son savoir dans l'action et dans la réflexion.

\section{Des questionnements pour conduire la réflexion sur la fonction enseignante}

Au Bénin, l'utilisation des enseignants communautaires remonte à 1986. Elle visait à faire face au gel du recrutement à la fonction publique alors que l'État marxiste-léniniste des années 1970-1980 était resté essentiellement l'unique employeur (Kakai, Lègba, Gansa, Tossou et Salami, 2008). Ce désengagement de l'État, renforcé depuis 2006 par les mesures de gratuité scolaire, notamment dans l'enseignement primaire, prises en marge des prévisions du Plan décennal de développement du secteur de l'éducation (PDDSE 2006-2015), a accentué les besoins en enseignants et conduit les parents d'élèves, en collaboration avec les écoles, à conclure des contrats avec des enseignants communautaires parmi les jeunes instruits du village. Cela a suscité l'inquiétude des autorités au regard de la faible qualité des apprentissages et a conduit l'État à imposer le BEPC (Brevet d'études du premier cycle) comme niveau minimum de recrutement de tout enseignant. Toutefois, dans la pratique, les indicateurs de qualité n'ont pas toujours été respectés : on a plutôt recherché celui qui ferait « office d'enseignant» plutôt que «l'enseignant professionnel» (STP-PDDSE, 2016). En somme, la professionnalisation des enseignants suppose la professionnalisation de leur formation. C'est sur cette base que l'on se propose d'appréhender les conséquences de l'utilisation des enseignants non qualifiés dans l'enseignement primaire au Bénin. Cependant, il faut retenir que si on peut faire l'hypothèse que l'utilisation des enseignants non qualifiés dits « communautaires » peut être un facteur de baisse de la qualité des apprentissages, il reste que le niveau académique desdits enseignants est assez variable, ce qui permettait d'observer une intervention différenciée selon les niveaux et par conséquent des effets tout aussi différenciés sur les apprentissages (Kakai, Lègba, Gansa, Tossou et Salami, 2008). Parfois, certains d'entre eux suivent après leur recrutement des formations qui leur permettent d'améliorer leur prestation, mais il s'agit souvent de formations "sur le tas", au contact du métier et sans le minimum de savoirs théoriques, contrairement aux formations dispensées dans les écoles de formation d'instituteurs qui portent sur des savoirs à la fois théoriques et pratiques.

Ces premiers développements amènent deux questions :

- la formation initiale apporte-t-elle effectivement une plus-value en matière de professionnalité et de qualité de l'enseignement-apprentissage?

- la présence d'enseignants communautaires influe-t-elle négativement sur la qualité de l'enseignement? 
La première question est d'autant plus pertinente qu'elle a été soulevée à plusieurs reprises dans différents documents de référence sans qu'on y trouve de réponse. Ainsi, en 1991, à la suite du diagnostic établi lors des États généraux de l'éducation; il a été suggéré « de repenser toute la politique de formation des professeurs d'Écoles normales et de personnes destinées à intervenir dans la formation initiale » (Actes des États généraux, 1991, p. 61). Plus tard en décembre 2014, les Actes du deuxième forum national sur le secteur de l'éducation relevaient pour l'enseignement primaire, la « faible pertinence de la formation dans les ENI » (Actes du deuxième forum national sur le secteur de l'éducation, 2015, p.74). Cela montre que, sur une période de 25 ans, les avancées en termes de formation dans les écoles normales sont peu visibles, d'où l'intérêt de remettre en question ici le dispositif de formation des futurs enseignants du primaire afin de savoir si les écoles de formation contribuent véritablement à la professionnalisation des enseignants. Il convient par ailleurs de remettre en question le contexte de la formation en général, ce qui nous amène à nous interroger sur le processus de formation continue. Les unités pédagogiques (UP) constituent le principal dispositif de formation continue. Elles ont pour objectif d'offrir à tous les enseignants l'occasion d'apprendre en situation professionnelle avec comme avantage les retours de leurs pairs sur leurs pratiques (Boko-vou, 2015; République du Bénin, 2013). Conformément à l'arrêté $\mathrm{n}^{\circ}$ 2013-324 portant attributions, organisation et fonctionnement des directions départementales des enseignements maternel et primaire, chaque zone pédagogique est organisée en unité pédagogique réunissant 3 ou 4 écoles en fonction du nombre d'écoles qui la composent. Chaque UP est dirigée par un responsable d'unité pédagogique et un coresponsable choisis par les directeurs d'écoles et les enseignants parmi les plus anciens considérés comme travailleurs et expérimentés (Boko-vou, 2015). Le responsable et le coresponsable jouent le rôle de formateurs.

Les résultats de l'évaluation de la maîtrise des contenus notionnels évoquée plus haut permettront de montrer que le recrutement d'enseignants communautaires n'est pas forcément le premier facteur à la base de la mauvaise qualité des enseignements. Le rapport d'étude intitulé Ce que je sais de ce que j'enseigne restitue les résultats d'évaluation de 3280 enseignants du primaire. Ces 3280 enseignants identifiés dans les six cours du primaire ont été observés en français et en mathématiques; 44,8\% des enseignants de cet échantillon sont des enseignants communautaires, 29,5\% sont des enseignants contractuels et $17,3 \%$ sont des agents permanents de l'État. Les épreuves de français et de mathématiques ont été conçues sur le modèle PASEC sous la forme de QCM (voir outil en annexe). Les épreuves de français ont couvert les domaines ci-après: la grammaire fonctionnelle, l'orthographe d'usage, la syntaxe des mots, le vocabulaire soutenu, les exercices d'appariement, les exercices à trous et les exercices de sémantique pour la détermination du sens des mots et l'expression. Les épreuves de mathématiques concernent les quatre opérations, à savoir l'addition, la soustraction, la multiplication, la division; des exercices de géométrie; des exercices de numération relatifs aux nombres complexes et aux fractions; des exercices de mesures relatifs aux mesures agraires; aux mesures de capacité et aux mesures de masse. Cette évaluation permet d'apporter des éléments de réponse à la deuxième question de recherche. 


\section{Méthodologie de la recherche}

Pour répondre à la première question de recherche, plusieurs catégories de données ont été mobilisées. D'abord, les données d'une enquête de terrain réalisée auprès de 53 élèves-maîtres des écoles publiques et privées de formation d'instituteurs permettront de mettre en exergue les difficultés d'ordre didactique et pédagogique rencontrées par ces derniers dans l'exercice de la fonction et la faible pertinence de la formation initiale. Ces élèves-maîtres ont été suivis lors de leur stage de professionnalisation dans des écoles primaires au cours de l'année 2015. L'enquête utilise une grille d'observation intitulée "Outil de suivi et d'évaluation pédagogique " (OSEP), composé de 16 critères regroupés en cinq domaines (cf. encadré) afin d'identifier globalement les compétences acquises par les enseignants. Dans le cadre du présent article, l'analyse est circonscrite à l'identification des habiletés développées par les élèvesmaîtres relativement au référentiel de compétences de la formation.

Domaine 1 : Gérer efficacement le temps d'enseignement/apprentissage, les techniques, les ressources, les connaissances.

1. Gère efficacement le temps d'enseignement.

2. Fait preuve d'habiletés et d'efficacité dans la gestion de la classe.

3. Utilise efficacement différentes ressources pédagogiques et stratégies d'enseignement pour construire des connaissances.

Domaine 2 : Créer un environnement d'apprentissage collaboratif et équitable.

4. Implique les élèves dans des activités d'apprentissage coopératif soigneusement organisées.

5. Met en œuvre un enseignement qui se fixe comme objectif le développement par les élèves d'habiletés sociales et collaboratives.

6. Fait participer activement tous les élèves aux activités d'apprentissage, quels que soient le sexe, le niveau de réussite, les acquis antérieurs, les aptitudes, les besoins spéciaux et les autres différences.

Domaine 3 : Promouvoir une ambiance où l'élève-maître est placé au centre de ses apprentissages, une ambiance qui privilégie des activités d'enseignement et des échanges.

7. Utilise des stratégies d'enseignement variées pour promouvoir une participation active de l'élève à son apprentissage.

8. Pose des questions ouvertes qui incitent les élèves-maîtres à la réflexion et facilitent l'explicitation de leur pensée.

9. Encourage les élèves-maîtres à s'exprimer librement quand ils se retrouvent dans l'environnement pédagogique.

Domaine 4 : Promouvoir un environnement pédagogique motivant, propice à la réflexion et à l'intégration des apprentissages.

10. Offre aux élèves des occasions d'établir des liens significatifs entre les résultats de leurs différents apprentissages et la vie courante.

11. Présente aux élèves des situations et des problèmes de la vie courante leur permettant de réinvestir les résultats de leurs apprentissages.

12. Donne aux élèves des occasions de réfléchir sur leurs démarches et sur les résultats de leurs apprentissages.

13. Engage les élèves dans un processus constructif de rétroaction.

14. Se réfère aux connaissances et aux expériences antérieures de ses élèves-maîtres pour planifier et pour ajuster au besoin les activités d'enseignement et d'apprentissage.

Domaine 5 : Promouvoir la résolution des problèmes, l'émulation et l'exercice de la pensée critique.

15. Offre aux élèves des occasions de faire des analyses et synthèses, de construire des preuves et de faire des déductions.

16. Donne aux élèves des occasions de résoudre des problèmes.

Figure 1

Présentation de l'OSEP. 
Les critères de l'OSEP sont évalués en fonction d'une échelle présentée dans le tableau 1.

\section{Tableau 1}

Pondération des critères de l'échelle d'appréciation.

\begin{tabular}{lr} 
Critères d'appréciation & Points attribués \\
\hline Décevant & 0 point \\
\hline Pas satisfaisant & 1 point \\
\hline Satisfaisant & 2 points \\
\hline Très satisfaisant & 3 points
\end{tabular}

Cette pondération permet d'attribuer aux différents domaines de l'OSEP les barèmes ci-après :

\section{Tableau 2}

Barème appliqué aux domaines de l'outil OSEP après pondération.

\begin{tabular}{|c|c|c|}
\hline $\mathbf{N}^{0}$ & Domaines & Barèmes \\
\hline I & Gérer efficacement le temps d'enseignement/apprentissage, les techniques, les ressources, les connaissances. & 9 points \\
\hline II & Créer un environnement d'apprentissage collaboratif et équitable. & 9 points \\
\hline III & $\begin{array}{l}\text { Promouvoir une ambiance où lélève-maitre est placé au centre de ses apprentissages, une ambiance } \\
\text { qui privilégie des activités d'enseignement et des échanges. }\end{array}$ & 9 points \\
\hline IV & $\begin{array}{l}\text { Promouvoir un environnement pédagogique motivant, propice à la réflexion et à l'intégration des } \\
\text { apprentissages. }\end{array}$ & 15 points \\
\hline V & Promouvoir la résolution des problèmes, l'émulation et l'exercice de la pensée critique. & 6 points \\
\hline Total & & 48 points \\
\hline
\end{tabular}

On suppose que le stagiaire qui obtient un score supérieur ou égal à 24 points est moyen et celui qui obtient moins de 24 points est faible.

Les 53 élèves-maîtres ont été observés en salle de classe de mathématiques et de français par les directeurs d'écoles, des conseillers pédagogiques et des inspecteurs. Il s'agissait de focaliser l'attention sur la démarche d'apprentissage et sur la démarche d'enseignement. Pour cela, l'observation en situation de classe n'était pas suffisante. Il fallait suivre un entretien dirigé par le conseiller pédagogique. Les résultats issus de l'analyse des données collectées grâce à l'outil OSEP permettent de comprendre que l'effet de la formation initiale est infime. L'analyse de contenu sémantique et syntaxique de l'entretien enregistré et transcrit laisse entrevoir une posture du formateur (le conseiller pédagogique) qui n'est pas toujours favorable à l'intériorisation des savoirs par l'élève-maître (Adékou et Baba-Moussa, 2017). 


\section{Présentation, analyse et interprétation des résultats de la recherche}

Les données traitées sur les 53 élèves-maîtres révèlent des faiblesses au regard des critères de cinq domaines retenus pour l'analyse. L'interprétation des données renvoie au constat d'insuffisances liées à la formation initiale qui ne semble pas apporter une plus-value au profit de ceux qui la suivent.

\section{Des résultats globalement faibles au regard des critères retenus pour les cinq domaines}

Les critères pour lesquels les élèves-maîtres sont véritablement faibles sont ceux situés dans la partie mise en exergue par le cercle. Moins de $50 \%$ des élèves-maîtres satisfont à ces critères.

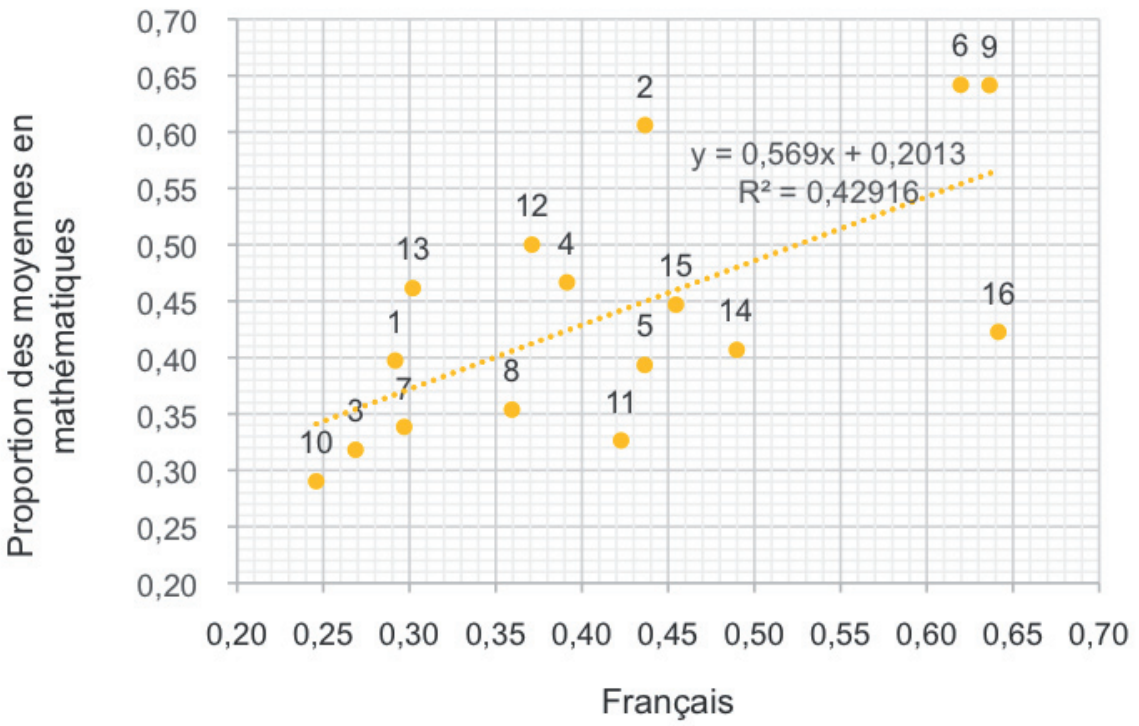

Figure 2

Distribution des critères OSEP.

Comme le montre la figure 2, la proportion d'élèves-maîtres qui réussit les critères 3-5-7-8-10-11-13 est très faible. Par ailleurs, on note chez les élèves-maîtres qui sont moyens dans différents domaines plusieurs difficultés au niveau des critères. Le développement qui suit résume pour chaque domaine les difficultés enregistrées lors des observations de classe.

Au niveau du domaine 1, on peut citer comme difficultés rencontrées la mauvaise utilisation des stratégies ou la non-variation des stratégies, une mauvaise programmation des activités qui ne correspond pas aux situations d'apprentissage, le non-maintien de la motivation des élèves, un défaut d'interaction soutenue entre maître et élève, une mauvaise formulation des consignes, une mauvaise utilisation des outils pédagogiques (moyens auditifs, audiovisuels). Tous ces aspects peuvent conduire à une mauvaise gestion du temps. 
Au sujet du domaine 2, il est réellement difficile pour les élèves-maîtres de communiquer efficacement. Au risque de s'exposer, les élèves-maîtres préfèrent poser des questions aux élèves plus éveillés. Cette attitude des élèves-maîtres porte entorse au principe de l'équité. Mais au-delà du problème lié à la communication, la difficulté pour les élèves-maîtres de se projeter dans le futur lors de la préparation de la situation d'apprentissage constitue un véritable frein en ce sens qu'ils n'arrivent pas vraiment à prévoir, à anticiper et à gérer. Il y a donc des difficultés de conception des activités qui s'ajoutent aux problèmes relatifs à la communication.

Pour le domaine 3, parfois l'élève-maître propose une stratégie inappropriée. Il peut avoir connaissance des différentes stratégies sans réellement les solliciter en temps opportun. L'élève-maître doit pouvoir encourager les élèves à s'exprimer. Certains se résignent à favoriser l'expression libre, soit en raison du risque auquel ils pourraient s'exposer face à des préoccupations des élèves auxquelles ils ne pourraient pas répondre ou en raison d'autres aspects que la recherche peut davantage creuser pour mieux comprendre le phénomène.

Pour le domaine 4, la confrontation des apprentissages à l'environnement des apprenants doit constituer une préoccupation de l'enseignant. Pouvoir rapprocher les connaissances disciplinaires des connaissances transdisciplinaires est l'intérêt principalement attendu ici dans ce critère. Les observations faites permettent d'estimer qu'il n'existe souvent aucune occasion de faire ce rapprochement. Seuls quelques élèves-maîtres utilisent les résultats de groupes comme des occasions propices permettant l'établissement de lien entre les apprentissages et la vie courante.

Enfin, pour le domaine 5, la difficulté pour les enseignants de repérer les perceptions des élèves ne leur permet pas également de faire le lien entre la situation et la vie courante. Pour réussir ce domaine, l'élève-maître doit proposer une mise en situation parfaite. Lorsque la mise en situation est réussie, les élèves éprouvent moins de difficulté selon les observateurs. Les élèves-maîtres pensent que ce domaine s'observe uniquement à la phase de retour et de projection d'une situation d'apprentissage.

La figure 2 présentée ci-dessus montre bien les difficultés rencontrées par les élèves-maîtres, observés à quatre mois de leur examen professionnel. Ces difficultés prouvent que la formation initiale n'a pas permis aux élèves-maîtres d'acquérir les compétences professionnelles nécessaires ou que les élèvesmaîtres n'acquièrent que très faiblement les compétences professionnelles exigées.

Au cours du stage de professionnalisation qui se déroule en deuxième année de formation, les élèvesmaîtres sont mis en responsabilité dans une classe à effectif variable. Ils reçoivent la visite des conseillers pédagogiques de façon périodique. À ce niveau également on constate que la posture du conseiller pédagogique n'a pas favorisé le développement de l'autonomie professionnelle.

\section{La posture du formateur comme frein à la construction des compétences professionnelles}

Les résultats de l'enquête auprès des 53 enseignants révèlent qu'en formation initiale, les élèvesmaîtres sont exposés à la prégnance des savoirs des formateurs transmis selon une démarche quasi impositive qui dicte les savoirs de la pratique tout comme si l'expérience des aînés était à rééditer. Or, l'expérience des aînés n'est pas forcément de l'expertise. Au cours des stages de professionnalisation, les conseillers pédagogiques adoptent une posture de "chef ", avec des conseils qui prennent souvent l'allure d'injonctions. Ainsi, lors du vécu pédagogique, après avoir observé plusieurs élèves-maîtres, le 
conseiller pédagogique les regroupe pour relever lui-même une à une leurs difficultés. Cette démarche ne s'identifie pas aux pratiques réflexives. Les pratiques réflexives exigent que l'élève-maître soit lui-même acteur de ses apprentissages et qu'il participe à identifier lui-même ses erreurs et même ses points forts. Dans l'extrait ci-dessous tiré des propos d'un conseiller pédagogique s'adressant à plusieurs élèves-maîtres, nous identifions plusieurs recettes émanant des savoirs de l'expérience. La démarche du $\mathrm{CP}$ est faite de constats séquencés par des appréciations de diverses natures. Ensuite, le $\mathrm{CP}$ propose ou impose sa démarche, celle dont il a connaissance qu'il étaye avec un argumentaire issu de son expérience. "[...] Aujourd'hui, la maîtresse a fait la lecture systématique. Elle est allée directement au manuel. Non! Partir d'abord de l'introduction. Comme vous connaissez vos enfants qui ne participent pas activement là. - Vous pouvez même partir d'une image - Là̀, vous voyez ceci là - ça ressemble à quoi? Et aujourd'bui, ab! Nous allons lire un texte hein qui va parler de quelque chose de comme ça... donc suivez! Vous allez observer les images. Vous allez lire le texte et imaginer le contenu du texte. Mais quand vous n'arrivez pas à lire le texte, vous allez faire quoi? (15'20). Si la maîtresse a l'babitude de faire ça, aujourd'bui, les stratégies de lecture vont sortir seulement de la bouche de ces enfants. Ces enfants ont soif de connaître, mais il y a quelques 2 ou 3 seulement qui animent et timidement la classe avec la maîtresse. Donc, rappelez chaque fois les stratégies, les petites stratégies de lecture dans les basses classes là aux enfants » (extrait de l'entretien du conseiller pédagogique, 2016).

Cet extrait comporte des interjections auxquelles a recours le $\mathrm{CP}$ soit pour dire que le futur enseignant fait fausse route (Non!), soit pour attirer l'attention du futur enseignant sur la formule proposée (ah) ou sur la formule qui peut marcher selon le CP. Comme pour proposer un style d'expression aux futurs enseignants qui devront à leur tour en faire usage dans leur classe pour, par exemple, rallier les élèves à leur cause, le CP utilise un ton flexible, voire adouci en guise d'exemple, un ton que le futur enseignant lui-même utilisera pour rallier les élèves à sa cause.

L'intervention des formateurs semble renvoyer ainsi, certes de façon caricaturale, aux pratiques des patrons d'ateliers artisanaux qui développent chez les apprentis un apprentissage par mimétisme des gestes du patron (Baba-Moussa et Flénon, 2014; Davodoun, 2014; Sidibé et Baba-Moussa, 2016). En conséquence, les élèves-maîtres ne développent pas tous une pratique réflexive et ne développent pas non plus les compétences attendues. En situation réelle de classe, ils sont confrontés à des problèmes d'ordre didactique, pédagogique et disciplinaire dans un système sans assistance qui les rend aussi bien vulnérables que responsables.

Cette faiblesse du mode de formation se double du faible niveau de maîtrise des savoirs professionnel par les élèves-maîtres. Le tableau 3 qui présente, en les croisant, les scores obtenus par les élèvesmaîtres à la suite des observations relatives à leur prestation en salle de classe pour l'enseignement du français (en ligne) et des mathématiques (en colonne) montre un faible pourcentage de réussite $(16,98$ \%) des élèves-maîtres des ENI à la fois en français et en mathématiques. En ce qui concerne les élèves des établissements privés de formation d'instituteurs (EPFI) qui réussissent à la fois en français et en mathématiques, ils sont 6 , soit 11,32 \% d'élèves-maîtres. Par contre, 32,08 \% d'élèves-maîtres des ENI échouent à la fois en français et en mathématiques contre $24,53 \%$ des élèves des EPFI. 


\section{Tableau 3}

Répartition des élèves-maîtres selon l'établissement et le niveau de réussite.

\begin{tabular}{lrrr}
$\begin{array}{l}\text { Niveau atteint en français par élèves } \\
\text { des ENI et des EPFI }\end{array}$ & Faibles en math & Moyens en math & Total général \\
\hline Élèves des ENI faibles en français & $\mathbf{1 7 ( 3 2 , 0 8 \% )}$ & $3(5,66 \%)$ & $20(37,74 \%)$ \\
Élèves des ENI moyens en français & $2(3,77 \%)$ & $\mathbf{9 ( 1 6 , 9 8 \% )}$ & $11(20,75 \%)$ \\
Total élèves des ENI appréciés en français & $19(35,85 \%)$ & $12(22,64 \%)$ & $31(58,49 \%)$ \\
Élèves des EPFI faibles en français & $\mathbf{1 3 ( 2 4 , 5 3 \% )}$ & $2(3,77 \%)$ & $15(28,30 \%)$ \\
Élèves des EPFI moyens en français & $1(1,89 \%)$ & $\mathbf{6 ( 1 1 , 3 2} \%)$ & $7(13,21 \%)$ \\
Total élèves des EPFI appréciés en français & $14(26,42 \%)$ & $8(15,09 \%)$ & $22(41,51 \%)$ \\
Total général des élèves appréciés en français & $33(62,26 \%)$ & $20(37,74 \%)$ & $53(100,00 \%)$ \\
\hline
\end{tabular}

On peut constater que plus de $50 \%$ (soit 32,08 \% des élèves maîtres des ENI et 24,53 \% des élèvesmaîtres des EPFI) d'élèves-maîtres des ENI et des EPFI ne réussissent ni l'enseignement du français ni l'enseignement des mathématiques. Les élèves-maîtres moyens dans les deux disciplines sont au nombre de 15, soit un peu plus de $25 \%$ de l'échantillon (c'est-à-dire 16,98 \% des élèves-maîtres des ENI et $11,32 \%$ des élèves-maîtres des EPFI).

Au regard de ces résultats, la formation initiale montre une valeur ajoutée assez modérée puisque les élèves-maîtres n'ont pas acquis un niveau de compétence suffisant dans les savoirs professionnels. La formation continue, cependant, n'est pas forcément pertinente pour relever ce défi. À ce niveau-là, les travaux menés par Boko-vou (2015), évoqués plus haut, révèlent des dysfonctionnements semblables à ceux de la formation initiale.

\section{L'absence d'une démarche d'ingénierie en formation continue : un véritable handicap au processus de construction des compétences professionnelles}

En analysant les résultats des élèves-maîtres évalués sur la base de l'Outil de suivi et d'évaluation pédagogique (OSEP) et qui n'atteignent pas les compétences requises pour enseigner; en analysant la posture du conseiller pédagogique, qui impose son savoir d'expérience aux élèves-maîtres au lieu de mettre en place un dispositif d'analyse des pratiques et en étudiant les résultats du dispositif de formation continue, notamment les unités pédagogiques, on convient que les futurs enseignants, les enseignants communautaires et même les enseignants qualifiés évoluent dans un environnement peu propice au développement de compétences professionnelles et à une réelle autonomisation professionnelle. Nous avons vu dans le développement précédent que la formation initiale produit des résultats peu satisfaisants. Cette section insiste sur l'environnement professionnel de l'enseignant qui, pour l'heure, n'est pas de nature à renforcer les compétences professionnelles des enseignants en général. En effet, le dispositif de la formation continue souffre de l'absence d'ingénierie de la formation. Or, il aurait fallu mettre véritablement en œuvre un dispositif efficace à partir de l'analyse réelle des besoins en formation des enseignants pour espérer une meilleure performance des enseignants. Selon les conseillers pédagogiques et les inspecteurs, l'organisation des unités pédagogiques relève d'abord de 
leur prérogative. Ils rédigent eux-mêmes les modules de formation en impliquant rarement les acteurs principaux qui sont entre autres les responsables d'unités pédagogiques, les enseignants et les futurs enseignants. Loin d'être une démarche savamment orchestrée par les formateurs, cette pratique de formation observée chez les formateurs est plutôt le signe de la méconnaissance totale du principe de l'ingénierie de la formation. Normalement, les responsables d'unités pédagogiques et les enseignants sont en droit de participer à l'identification de leurs propres besoins en formation afin de s'identifier à travers les thématiques qui seront développées lors des formations. Mais les inspecteurs et les conseillers pédagogiques considèrent qu'il leur échoit particulièrement le rôle de produire le savoir qui sera mis à la disposition des responsables d'unités pédagogiques chargés à leur tour d'exécuter ces modules. Dans une telle dynamique, l'objectif visé, qui est la formation de l'enseignant à la réflexivité, est détourné.

Dès lors que les dispositifs de formation continue comportent des insuffisances qui les rendent inopérants, on peut en déduire que les enseignants débutants et/ou communautaires ne bénéficient pas vraiment d'un dispositif de formation favorisant l'accès à l'autonomie dans les prises de décisions. Or, il s'agit là d'une condition importante de la professionnalisation des enseignants puisque « l'acquisition des compétences n'est possible que s'il existe une reconnaissance et une intériorisation par l'apprenant des observations qu'ils reçoivent en coaching »(Becker, 2004, p. 6). Cette dimension n'est valorisée ici ni en formation initiale ni en formation continue des enseignants. Si la présence des enseignants communautaires augmente le besoin en formation, en revanche, elle n'est pas forcément la source principale de la déprofessionnalisation de la fonction enseignante.

Malgré le statut de l'enseignant communautaire, sa prestation en salle de classe n'est pas pour autant alarmante lorsqu'on la compare à celle des plus anciens enseignants. En faisant référence à l'évaluation des contenus notionnels, toute suspicion disparait au profit d'une cote particulière accordée à cette catégorie d'enseignants. Cette évaluation permet de constater que l'ancienneté n'est pas une variable qui discrimine les enseignants et que les enseignants communautaires n'ont pas toujours démérité. Autrement dit, les plus anciens ne sont pas forcément les meilleurs. Au contraire, ils sont parfois moins bons que les plus jeunes (Bahna et Houédo, 2015). Il existe donc un malaise profond qui n'est pas lié au recrutement des enseignants communautaires, mais plutôt à l'absence d'engagement de la part de l'enseignant. Cet engagement, qui est possible à travers une réelle implication du professionnel dans le processus de formation continue, peut constituer une réelle source motivationnelle et un point d'ancrage fort pour la construction de l'autonomie professionnelle.

\section{Conclusion}

Les dysfonctionnements de la formation initiale et continue ne permettent pas le développement harmonieux des compétences professionnelles. Si la formation initiale effectuée en deux ans ne comble pas les attentes, on peut s'attendre à ce que la formation continue joue le rôle de relais pour combler les éventuelles lacunes. Les constats de terrains effectués dans le cadre des études citées plus haut relèvent des blocages au niveau opérationnel, hypothéquant ainsi la valeur ajoutée que devrait avoir la formation en unité pédagogique. On retient en somme un effet assez modéré de la formation (formation initiale et formation continue) sur les enseignants et notamment sur les élèves-maîtres. 
Les avancées en matière de professionnalisation sont ainsi freinées par l'absence d'autonomie (même relative) des enseignants qui ne sont pas en mesure d'agir de façon efficace en situation. Le présent article a permis de montrer que les enseignants en général ont des difficultés aussi bien au niveau des compétences professionnelles que des compétences disciplinaires et que l'amélioration des dispositifs de formation pourrait contribuer à l'amélioration de la qualité des enseignements. En revanche, la stigmatisation d'une catégorie d'enseignant, dans le contexte actuel de formation, réduirait la portée des actions novatrices qui doivent, pour être efficaces, prendre en compte les besoins de tous les enseignants du Bénin.

\section{Références}

Adékou, C. (2017). «Professionnalisation » ou "professionnalisations»? Le paradoxe de la formation dans les écoles normales et établissements privés de formation d'instituteurs au Bénin (Thèse de doctorat inédite). Université d'Abomey-Calavi.

Adékou, C. et Baba-Moussa, A. R. (2017). Savoirs d'expérience et culture de la professionnalité dans la formation des enseignants de l'école primaire au Bénin. Transmettre, 1(4), 129-139. Repéré à http://bec.uac.bj/uploads/publication/9cca9f9649ec0ffa7dbfc24f3b556f5c.pdf

Altet, M. (2014). Formes de résistance des pratiques de formation d'enseignants à la pratique réflexive et conditions de développement de la réflexivité. Dans M. Altet, J. Desjardins, R. Étienne, L. Paquay et P. Perrenoud, Former des enseignants réflexifs (p. 39-59). Bruxelles : de Boeck.

Altet, M. (2015). Les compétences de l'enseignant-professionnel : entre savoirs, schèmes d'action et adaptation, le savoiranalyser. Dans M. Altet, J. Desjardins, R. Étienne, L. Paquay et P. Perrenoud, Former des enseignants réflexifs (p. 4357). Bruxelles : de Boeck.

Attanasso, M. O. (2010). Bénin - Prestation efficace des services publics de l'éducation. Repéré à https://fr.scribd.com/document/80789087/Benin-Education-sector-FRENCH-Web-Benin

Baba-Moussa, A. R. (2006). Problématique de la crise de l'éducation en Afrique francophone subsaharienne. Dans Actes des assises francophones de l'éducation et de la formation (p. 229-240). Paris: Organisation internationale de la Francophonie.

Baba-Moussa, A. R. (2017). La prise en compte de la relation entre formation et emploi dans la réforme de l'éducation au Bénin : contribution à l'élaboration d'un nouveau modèle éducatif. International Review of Education, 63(5), 631-656. http://dx.doi.org/10.1007/s11159-017-9659-9

Baba-Moussa, A. R. et Flénon, A. (2014). L'apprentissage artisanal, un contexte d'action éducative non formelle. Les stratégies d'orientation des apprentis au Bénin. Éducation permanente, (199), 93-104.

Bahna, A. H. et Houédo, V. F. (2015). Évaluation de la maîtrise des contenus notionnels par les enseignants : "Ce que je sais de ce que j'enseigne». Rapport d'évaluation. Document inédit.

Boko-vou, E. C. (2015). Évaluer la formation en unité pédagogique et proposer une démarche de mise en cuvre à visée de professionnalisation (Mémoire de master). Université de Lille.

Bourdoncle, R. (1993). La professionnalisation des enseignants : les limites d'un mythe. Revue française de pédagogie, (105), 83-119. http://dx.doi.org/10.3406/rfp.1993.1283

Davodoun, C. (2014). L'apprentissage des métiers artisanaux. Dans Actes des ateliers thématiques en préparation au deuxième forum national de l'éducation (vol. 2, p. 525-556).

Kakai, A. G., Lègba, R., Gansa, B., Tossou, R. et Salami, N. D. (2008). Le métier d'enseignant communautaire : profil de l'éducateur et impact sur le système éducatif béninois. Cotonou : ROCARE.

Maingari, D. (1997). La professionnalisation de l'enseignement au Cameroun. Des sources aux fins. Recherche et formation, (25), 97-112. http://dx.doi.org/10.3406/refor.1997.1432 
Paquay, L. (2015). Continuité et avancées dans la recherche sur la formation des enseignants. Avant-propos de la 4 édition. Dans M. Altet, J. Desjardins, R. Étienne, L. Paquay et P. Perrenoud (dir.), Former des enseignants réflexifs (p. 6-26). Bruxelles : de Boeck.

Perrenoud, P. (1996). Le métier d'enseignant entre prolétarisation et professionnalisation : deux modèles du changement. Perspective, 26(3), 543-562.

République du Bénin. (2013). Plan décennal de développement du secteur de l'éducation actualisé. Phase 3/2013-2015. Cotonou, Bénin.

République du Bénin. (2015). Actes du deuxième forum national sur le secteur de l'éducation. Cotonou, Bénin.

STP-PDDSE. (2016). Rapport de la première revue sectorielle de l'éducation - avril 2016, Cotonou, STP-PDDSE.

Wittorski, R. (2008). La professionnalisation. Savoirs, (17), 11-38.

\section{Pour citer cet article}

Adekou, C. et Baba-Moussa, A. R. (2019). La formation initiale et continue au Bénin : entre professionnalisation et déprofessionnalisation. Formation et profession, 27(1), 20-36. http://dx.doi.org/10.18162/fp.2018.442 


\section{Annexe}

Outil d'évaluation de la langue française (45 min) dans le cadre de l'étude intitulée « ce que je sais de ce que j'enseigne»

\section{Texte d'appui : l'Égypte est un don du Nil}

La civilisation égyptienne naît il y a environ 5000 ans dans la vallée et le delta du Nil. Le Nil est le plus long fleuve d'Afrique. Il coule du sud vers le nord et se jette dans la mer Méditerranée. Il prend sa source dans la région des Grands Lacs en Afrique de l'Est. L'Égypte est un pays désertique où l'installation des hommes aurait été impossible sans la présence du Nil.

À cette époque, la majorité des Égyptiens vivaient le long du fleuve. Ils chassaient, pratiquaient la pêche et cueillaient le lotus et le papyrus sur ses rives. Chaque année, de juillet à décembre, le Nil entrait en crue, ce qui provoquait une inondation importante de la vallée. Pendant sa décrue de juin à mai, le Nil déposait sur les terres qui le bordent une boue fertile comme un engrais, appelée limon. Le Nil était même considéré comme un Dieu par les Égyptiens, car il apportait la vie.

En 1970, un grand barrage a été construit sur le Nil. Depuis sa construction, le barrage permet de diminuer les inondations et garantit l'eau courante et l'électricité à tous. Les paysans peuvent maintenant réaliser plusieurs récoltes par an et cultiver plus de terre pour mieux nourrir la population.

Consigne : Réponds aux questions de 1 à 10 en cochant la case de la bonne réponse.

\begin{tabular}{|l|l|l|l|l|l|}
\hline \multicolumn{2}{|l|}{ 1. Qu'est-ce que le Nil? } & & \multicolumn{2}{|l|}{ 2. Il y a $\mathbf{0 0 0}$ ans, la plupart des Égyptiens vivaient... } \\
\cline { 1 - 2 } & A. & Le plus long fleuve d'Afrique & & dans la région des Grands Lacs \\
\hline B. & Un papyrus égyptien très ancien & & B. & & le long du fleuve \\
\hline C. & Une boue fertile d'un fleuve & C. & & dans des maisons en lotus et en papyrus \\
\hline D. & Un dieu égyptien d'un fleuve & & D. & & dans les pyramides des Égyptiens \\
\hline
\end{tabular}

\section{Chaque année, le limon déposé par le Nil permet de...}

\begin{tabular}{|c|c|}
\hline A. & construire des maisons. \\
\hline B. & cueillir le lotus et le papyrus. \\
\hline C. & cultiver les champs. \\
\hline D. & chasser et pêcher. \\
\hline
\end{tabular}

\begin{tabular}{|l|l|l|}
\hline \multicolumn{3}{|l|}{ 4. L'Égypte est un don du Nil, car... } \\
\hline A. & & le Nil se jette dans la Méditerranée. \\
\hline B. & & l'Égypte est un pays désertique. \\
\hline C. & & la civilisation égyptienne a environ 5000 ans. \\
\hline D. & & le Nil apporte l'eau et la vie à l'Égypte. \\
\hline
\end{tabular}

\begin{tabular}{|c|c|c|c|}
\hline \multicolumn{2}{|c|}{$\begin{array}{l}\text { 5. «L'Égypte est un pays désertique ». Je mets cette } \\
\text { phrase au plus-que-parfait : }\end{array}$} & \multicolumn{2}{|c|}{$\begin{array}{l}\text { 6. « Le Nil est le plus long fleuve d'Afrique ». Cette } \\
\text { phrase est : }\end{array}$} \\
\hline A & L'Égypte avait été un pays désertique. & A. & une proposition principale \\
\hline$B$ & L'Égypte sera un pays désertique & B. & une proposition indépendante \\
\hline$C$ & L'Égypte a été un pays désertique & C. & une proposition subordonnée relative \\
\hline$D$ & L'Égypte eut été un pays désertique. & $D$. & une proposition conjonctive \\
\hline
\end{tabular}




\begin{tabular}{|l|l|l|l|l|l|l|}
\hline \multicolumn{2}{|l|}{ 7. Je donne un paronyme du mot « barrage ». } & & \multicolumn{2}{|l|}{$\mathbf{8 .}$ « Le barrage permet de diminuer les inondations », cela veut dire que : } \\
\cline { 1 - 1 } & A. & démarrage & A. & & le barrage permet d'annuler les inondations. \\
\hline B. & mariage & B. & & le barrage permet de supprimer les inondations. \\
\hline C. & garage & C. & & le barrage permet de modifier les inondations. \\
\hline D. & bavardage & D. & & le barrage permet de réduire les inondations. \\
\hline
\end{tabular}

\begin{tabular}{|c|c|}
\hline \multicolumn{2}{|c|}{ 9. Dans ce texte, j'identifie des terminaisons. } \\
\hline A. & Méditerranée, terminaison « e » \\
\hline B. & paysans, terminaison «s» \\
\hline C. & population, terminaison « $\mathrm{n} »$ \\
\hline D. & juillet, terminaison « $t »$ \\
\hline
\end{tabular}

\begin{tabular}{|l|l|l|}
\hline \multicolumn{2}{|l|}{ 10. Le barrage construit sur le Nil en 1970 est : } \\
\hline A. & & un ouvrage économique. \\
\hline B. & & un instrument de pêche. \\
\hline C. & & un outil agricole. \\
\hline D. & & un don du Nil. \\
\hline
\end{tabular}

Note : nous ne présentons qu'un seul outil. Il y a d'autres outils qui ont servi à mesurer les connaissances des enseignants. 